\title{
Análisis de valoración contingente de restauración ecológica de una cantera en Soacha, Cundinamarca, Colombia
}

\section{Analysis of contingent valuation of ecological restoration of a quarry en Soacha, Cundinamarca, Colombia}

\section{Análise de valoração contingente de restauração ecológica de uma pedreira em Soacha, Cundinamarca, Colômbia}

\author{
Lina Melissa Tequia Mayorga' \& David Andrés Camargo Mayorga² \\ ${ }^{1} E$ Ecóloga. ${ }^{2}$ Economista, Magister en Educación.
}

${ }^{1}$ Pontificia Universidad Javeriana. ${ }^{2}$ Docente de Tiempo Completo de la Facultad de Ciencias Económicas. Miembro del Grupo de Estudios Contemporáneos en Contabilidad, Gestión y Organizaciones.

Universidad Militar Nueva Granada. Bogotá. Colombia

1melitequia@gmail.com, ${ }^{2}$ david.camargo@unimilitar.edu.co

\section{Resumen}

La investigación tuvo como objetivo analizar la disponibilidad a pagar por la realización de la restauración ecológica de la cantera Cueva del zorro, por parte de los habitantes de la vereda Panamá, en el municipio de Soacha, Cundinamarca, Colombia. Metodológicamente se aplicaron encuestas y una entrevista al líder comunitario de la comunidad con el fin de conocer la percepción acerca del ambiente, la restauración y la minería. La información recopilada fue analizada a través de estadísticas descriptivas. Los resultados muestran que los niveles socioeconómicos de la población influyen directamente en la toma de decisiones en cuanto al valor monetario ofrecido para la realización de la restauración.

Palabras clave: economía ambiental, ecología, minería, disponibilidad a pagar.

\begin{abstract}
The research had as objective analyze the availability to pay by the realization of the restoration ecological of the quarry Cave of the Fox, from them inhabitants of the sidewalk Panama, in the municipality of Soacha, Cundinamarca, Colombia. Methodologically were applied surveys and an interview to the leader community from the community to know the perception about the environment, the restoration and the mining. The information collected was analyzed through descriptive statistics. The results show that the socio-economic levels of the population directly influence decisions regarding the monetary value offered for the completion of the restoration.
\end{abstract}

Key-words: environmental economics, ecology, mining, availability to pay. 


\section{Resumo}

A pesquisa teve como objetivo analisar a disponibilidade de pagamento pela realização da restauração ecológica da pedreira Cueva del zorro pelos habitantes do local Panamá, em Soacha, Cundinamarca, Colômbia. Seguindo a metodologia, foram feitas pesquisas e uma entrevista ao líder comunitário da comunidade com a finalidade de conhecer a percepção sobre o ambiente, a restauração e a mineração.
A informação recolhida foi analisada usando estatística descritiva. Os resultados mostraram que níveis socioeconómicos da população influenciam diretamente nas decisões enquanto ao valor monetário oferecido para a realização da restauração.

Palavras-chave: economia ambiental, ecologia, mineração, disponibilidade de pagamento.

\section{Introducción}

Cada vez más son las exigencias en cuanto a regulación, control, composición y corrección de los impactos que pueda generar cualquier plan, obra o actividad, lo que incluye la minería, por lo que las comunidades se inquietan ante la posibilidad de cualquier cambio en su bienestar; motivo por el cual distintas disciplinas tienen como objetivo estimar los costos y beneficios no vislumbrados por los planes, obras y actividades; mediante técnicas como la valoración contingente, incluida dentro de la rama de la economía ambiental (Grajales, s.f.).

La degradación y destrucción de ecosistemas a nivel global, debido a diferentes factores ha acelerado la crisis ambiental en los últimos años, ya que se ven reducidos los múltiples servicios ambientales que prestan los ecosistemas, como por ejemplo la producción de agua, fijación de $\mathrm{CO}_{2}$, suelos productivos, biodiversidad, coberturas vegetales que evitan la erosión. Los índices de destrucción de los ecosistemas permanecen aceleradamente producido por prácticas agrícolas, ganaderas, industriales y de explotación no sostenible (Vargas \& Reyes, 2011).

Debido a la constante necesidad del ser humano por crear un entorno con una mejor infraestructura, se ha generado un deterioro de los ecosistemas que va en aumento, debido a la explotación directa que existe sobre estos. Diversas son las actividades que causas estos problemas sobre los ecosistemas; un claro ejemplo de estas es la minería, la cual tiene efectos tanto directos como indirectos; entre los primeros podemos encontrar la remoción de suelo, considerado el componente más afectado en la explotación minera , la pérdida de cobertura vegetal cambios físico-químicos del sustrato; entre los segundo podemos encontrar la interrupción o perdida de flujos energéticos - ecológicos y el aumento en el riesgo de deslizamientos, lo cual representa un riesgo para la sociedad (Rozo, 2007).

Las canteras son un tipo de actividad minera a cielo abierto que elimina en su totalidad los componentes de los ecosistemas como son la cobertura vegetal, la fauna y el suelo, además de alterar las condiciones microclimáticas de las zonas en donde se ha realizado la actividad. La minería ha tenido un crecimiento acelerado en el último periodo de tiempo (Fedesarrollo, 2008). En América Latina y en el caso específico de Colombia ha tenido una gran inversión por parte de grupos empresariales extranjeros, debido a que la minería es rentable y aporta empleos e impuestos al país que la acoge (Vicente et al., 2011), conllevando a un crecimiento económico y al desarrollo en términos de infraestructura.

En el caso particular del municipio de Soacha, Cundinamarca, Colombia, esta actividad se realiza 
con fines de extracción de materiales pétreos para la construcción de infraestructura: centros comerciales, carreteras, viviendas, entre otras; Soacha está clasificada como un área con aptitud para la explotacion; existen 5 veredas en las cuales se realiza esta actividad .panamá, el charquito, fusunga, alto de la cabra y alto de la cruz-, en esta área se encuentran arseniscas, cuarzosas de grano fino y piedra zonga las cuales se denominan como la formación Guadalupe, las cuales cuentan con afloramientos rocosos del Cretácico superior. Dichos espacios están ubicadas en zona de faIla, lo cual facilita la explotacion debido al grado de fracturación presente en las rocas (Alcaldía de Soacha, s.f.).

Teniendo en cuenta que Soacha es un municipio en constante crecimiento $4.8 \%$, superior a la media nacional del $1.7 \%$ y a Bogotá con el $2.1 \%$ (DANE, 2003), la demanda de infraestructura se hace más urgente e intensa, adicional a esto se debe tener en cuenta que dicha actividad de explotación no es exclusiva para el uso y empleo en el municipio, ya que es transportado y utilizado en las zonas aledañas, como Bogotá, implicando volúmenes aún mayores de extracción y por consiguiente mayores impactos y repercusiones, tanto sociales como ambientales. La falta de implementación de acciones y planes de manejo para estas áreas degradadas conlleva a que en el momento del abandono de la cantera se generen problemas de índole social, de salud, de seguridad etc. (Milgrom, 2007); para evaluar los efectos existen metodologías alternativas como lo es la valoración contingente -MVC-, la cual es una herramienta o método para estimar el valor de bienes y servicios, para los cuales no se ha generado un valor en el mercado basándose en preguntas direccionadas a los actores principales.

Herramientas como la valoración contingente son de gran valor debido a que reflejan el interés de los actores por recuperar o mantener un bien o servicio que está siendo afectado. La MVC busca estimar la máxima disposición a pagar de un individuo por el mantenimiento o mejora de un bien de no mercado o, la mínima disposición a ser compensado por la pérdida o disminución del disfrute del mismo bien (Kristrom \& Riera, 1994). Para la comunidad es importante tener información tangible que demuestre el valor del ambiente, solo entonces valdrá la pena conservarlo, cuidarlo y darle un manejo consiente. Eso acerca a la sociedad a decidir cuánto están dispuestos a sacrificar para conservar el ambiente y los beneficios que este les genera (Tomasini, s.f). Por tanto, la intención de esta investigación es tener una perspectiva de las posturas tanto de los propietarios, como de los trabajadores, compradores y la misma comunidad, frente al caso de la restauración de la cantera Cueva del zorro del municipio de Soacha, Cundinamarca, Colombia.

\section{Metodología}

El área de estudio se encuentra situada al sur del municipio de Soacha, la vereda Panama, está ubicada adyacente a la vía que conduce a la vereda de Fusungá y es una de las zonas rurales de este municipio, en dicha vereda está ubicada la cantera Cueva del zorro (Ver Figura 1). La cantera está en un predio de 10.5919 ha, y se encuentra dentro de las cotas de 2.614 msnm y 2.730 msnm. Hidrográficamente los drenajes de importancia en el área de estudio conducen sus aguas hacia la red de alcantarillado y aguas Iluvias de Soacha, siendo la más importante la que corresponde a la cuenca del Río Soacha. Soacha es uno de los municipios colombianos que presenta un mayor grado de desorden físico, espacial y ambiental; con importantes problemas en términos sociales, laborales, de seguridad y de contaminación (AIcaldía de Soacha, 2012). 


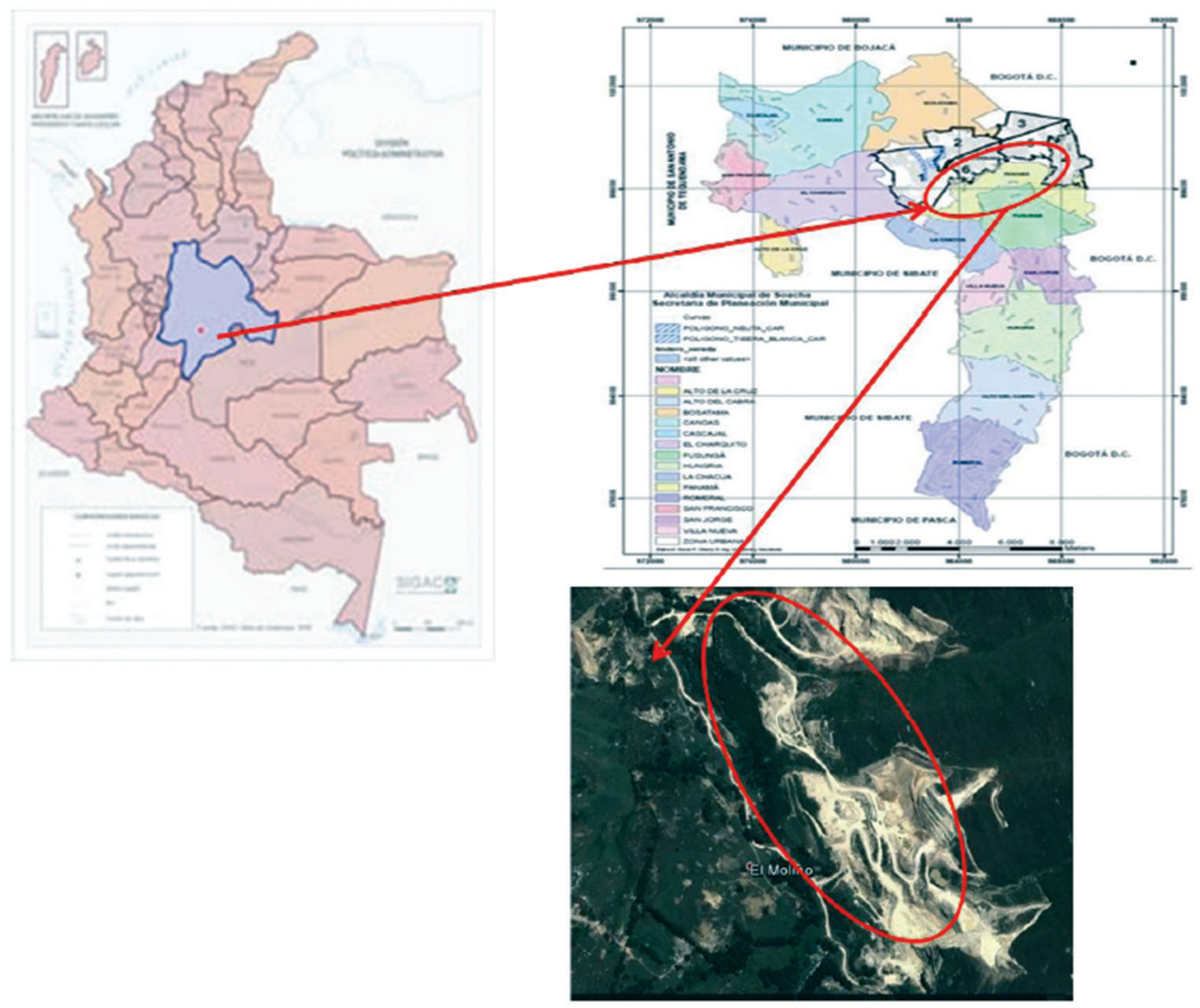

Figura 1. Localización geográfica de la vereda panamá en el municipio de Soacha Cundinamarca, Colombia.

Fuente: Modificado por los autores de http://www.soacha-cundinamarca.gov.co/mapas_municipio.shtml, y Google Earth para imagen satelital de la cantera Cueva del zorro.

La orientación metodológica de esta investigación es analítica explicativa, con herramientas cualitativas y cuantitativas, en donde se indaga la disponibilidad a pagar por la restauración ecológica por parte de la comunidad afectada por la actividad minera de la vereda panamá en el municipio de Soacha, Cundinamarca, Colombia. Se llevaron a cabo tres fases principales (Figura 2). La fase preliminar en donde se compilo información bibliográfica clave, acerca de temas como la valoración contingente y la restauración ecológica de canteras, en esta fase también se hizo reconocimiento del área de estudio por medio de la observación directa. Posteriormente se inició la fase de campo en la cual se realizaron 5 salidas al área de estudio, en las cuales se desarrollaron 100 encuestas aleatorias a habitantes en la zona de influencia directa de la cantera Cueva del zorro, para elegir el numero representativo de muestra se tuvo en cuenta la población inmediata al área de estudio, es decir aquella que se ve afectada directamente e inmediatamente por las actividades mineras, y una entrevista al líder comunitario de la vereda. Finalmente, se tuvo la fase de análisis, en la cual se presentaron los datos y se analizaron, además de realizarse el debido análisis de estos por medio de pruebas estadísticas mediante el uso del programa Stata 10.0. 


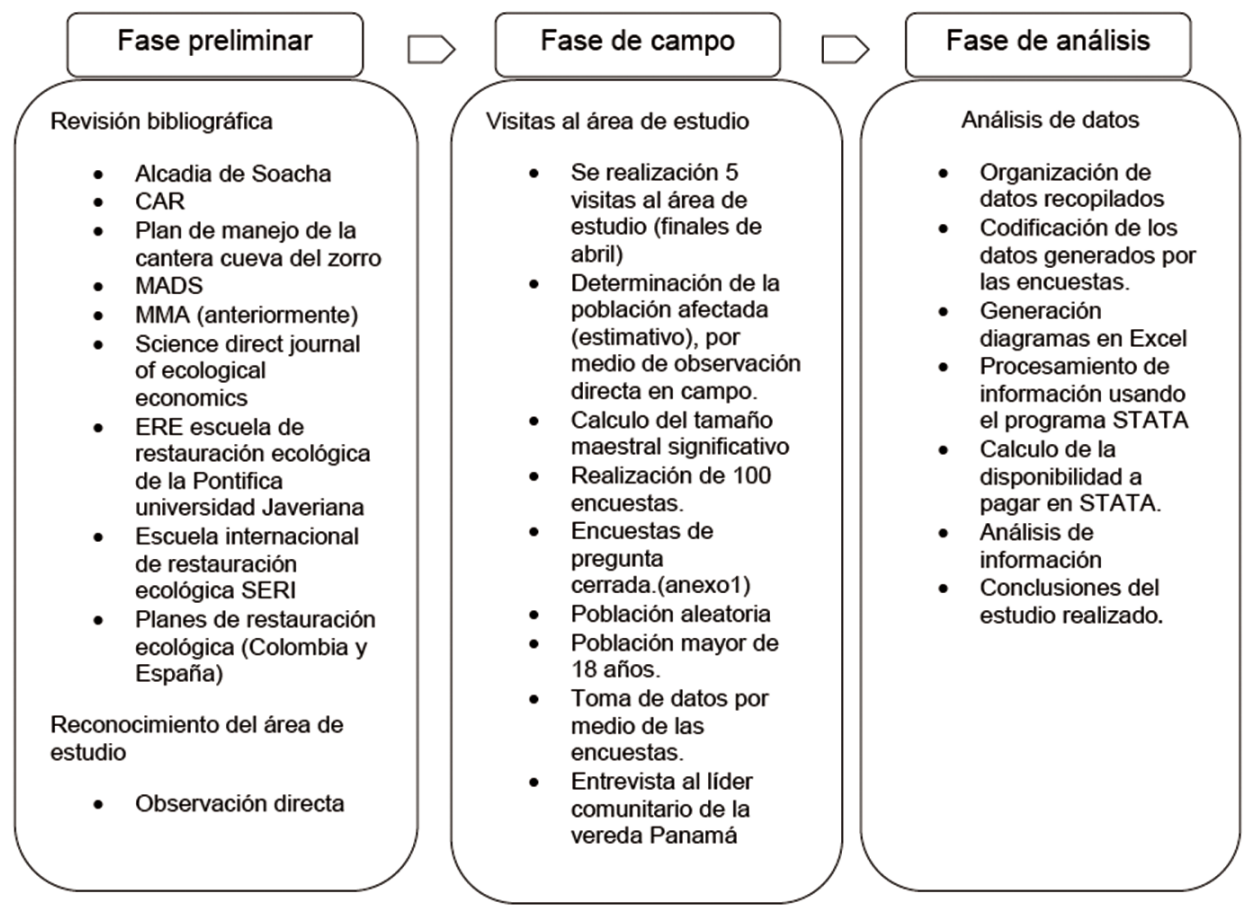

Figura 2. Diagrama metodológico de las fases de la investigación.

Se implementó la metodología con base en la bibliografía revisada, la información primaria se tomó directamente en el área de estudio por medio de 5 visitas a la vereda Panamá en Soacha, se usaron herramientas cuantitativas y cualitativas dentro de las primeras se realizaron encuesta y para las segundas se realizó una encuesta al líder comunitario de la vereda panamá. Para las encuestas la muestra se eligió aleatoriamente; esta debía cumplir con el criterio cuantitativo y cualitativo, el primero indica que el número debería ser significativo para el estudio, y el segundo debe presentar exactamente las mismas cualidades y características de la población de la cual se extrae (Downie \& Heath, 1983; De la Rosa, 2008) para lograr esto se realizó una observación directa para identificar la población afectada inmediatamente.

Según datos del Sisben (2013) la vereda Panamá presenta una población de aproximadamente 400 habitantes; mediante la fase de campo se realizó una observación directa y un dialogo con algunas personas para obtener información clave acerca de la población afectada, de lo cual se estipulo que la población afectada era alrededor de 200 personas, debido a que estas son las personas que viven alrededor de la cantera, y las cuales se ven mayormente afectadas. A partir de esto se realizó el análisis estadístico para hallar el número muestral representativo, dando como resultado el tamaño muestral significativo como 132 individuos, pero solo fue posible la realización de 100 encuestas, debido a la disposición de las personas por lo cual también se recurrió a la realización de dos entrevistas a los líderes comunitarios de la vereda Panamá; estas encuestas fueron domiciliares y se realizaron a cada persona cabeza de hogar, y de esta forma captar a su vez la información general de la familia, y tomar la posición de este individuo como la del hogar, abarcando de esta forma un número mayor de opiniones, respecto al tamaño muestral. Teniendo en cuenta el estado civil de casados de la gran mayoría de individuos, y el número de hijos y familiares con los que viven; el estudio abarcó más de 200 opiniones diferentes, sobrepasando de este modo el tamaño significativo de la muestra.

La información obtenida de las encuestas realizadas, se tabuló y por medio de estadística descriptiva, 
enfrentando la variable dependiente en este caso DAP frente a las demás variables (Tabla 1) se analizó mediante el programa estadístico STATA (1985), para evaluar la disponibilidad a pagar sobre la restauración ecológica de la cantera Cueva del zorro por parte la población encuestada.

Tabla 1. Descripción de variables utilizadas en la encuesta.

\begin{tabular}{l|l}
\hline \multicolumn{1}{c}{ Variable } & \multicolumn{1}{c}{ Explicación } \\
\hline Edad & Años del individuo \\
\hline Nivel de escolaridad & Años de educacion terminados \\
\hline Dap (disponibilidad a pagar) & Eleccion entre si y no de acuerdo a su disposicion a pagar \\
\hline Importancia del ambiente & Perspectiva del individuo hacia el medio ambiente. \\
\hline Nivel de ingresos & Ingresos personales netos mensuales \\
\hline Actividades mas dañiñas & Actividades que causan mayor impacto en de municipio de Soacha \\
\hline Valor dado & $\begin{array}{l}\text { Valor monetario dado por el individuo, respecto a la disposicion } \\
\text { a pagar por la restuaracion ecologica. }\end{array}$ \\
\hline
\end{tabular}

La encuesta se basó en preguntas de tipo cerrado, el cuestionario usado fue de tipo directo debido a que la población encuestada completo el formato personalmente; estas encuestas fueron realizadas en la vereda panamá en el municipio de Soacha, se diligenciaron casa por casa en la zona delimitada como zona de influencia directa.

La entrevista realizada fue semi-estructurada, lo cual indica que esta compuestas por puntos definidos pero estos van tomando mayor o menor profundidad a medida que se va realizando el dialogo con el entrevistado, pero aun asi presenta una estructura estandarizada por su forma y orden y a que abordan el mismo tema.

\section{Resultados}

\section{Percepción del ambiente}

Respecto a la percepción que tienen los encuestados habitantes de la vereda Panamá en el municipio de Soacha, por medio de la realización de las encuestas. Se obtuvo que el $86 \%$ de los encuestados considera importante el cuidado del medio ambiente, frente a un $14 \%$ que no considera importante cuidar el medio ambiente (Figura 3)

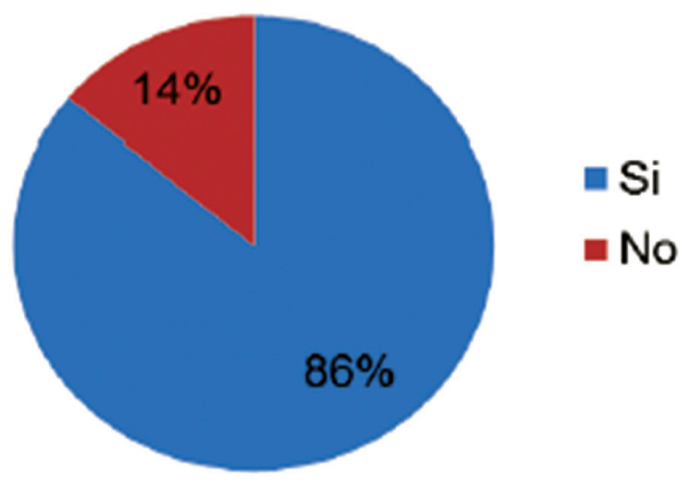

Figura 3. Percepción de la importancia del ambiente por parte de los encuestados.

En la Figura 4 se evidencia la decision de los encuestados acerca de las actividades que consideran mas dañiñas para el medio ambiente en el municipio de soacha cundinamarca, de 4 opciones dadas en la encuesta estos elegieron dos, de estas dos la mineria obtuvo un valor de $76 \%$, frente a las fabricas e industrias con un valor de $24 \%$. 


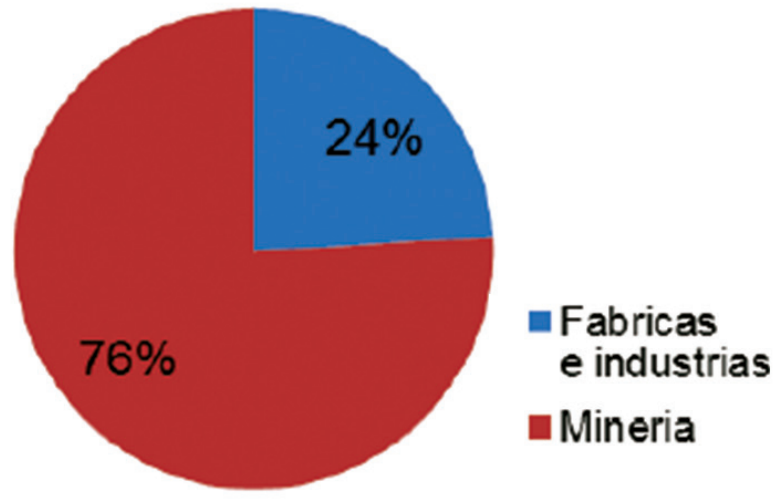

Figura 4. Actividades mas dañinas para el medio ambiente.

A los encuestados se les cuestionó acerca de la necesidad de restaurar las areas degradadas por la mineria realizada por la cantera Cueva del zorro de lo cual se evidencio que el $16 \%$ consideran que no es importante realizar una restauración en dichas areas, y un $84 \%$ que razonan que es importante darle una restauracion a las areas restauradas (Figura 5).

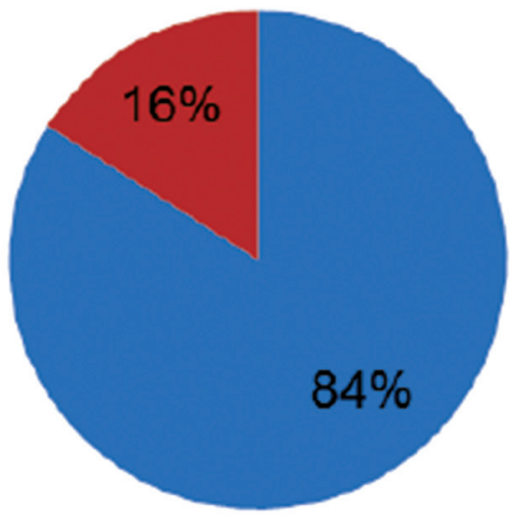

$\square \mathrm{Si}$

No

Figura 5. Necesidad de restauracion para areas degradadas de la cantera Cueva del zorro.

\section{DAP Disponibilidad a pagar}

De acuerdo a los datos obtenidos por medio de las encuestas realizadas se enfrentaron diversas variables con a la disponibilidad a pagar por la restauración ecológica de la cantera Cueva del zorro, de lo cual se obtuvo lo siguiente:

En relación a la proporción de género de los encuestados, dio como resultado que de 44 mujeres en el estudio, solo 4 (9\%) tienen la disposición a pagar por la restauración ecológica; por su parte de los 56 hombres 14 (25\%) tiene la disposición a pagar (Figura 6).

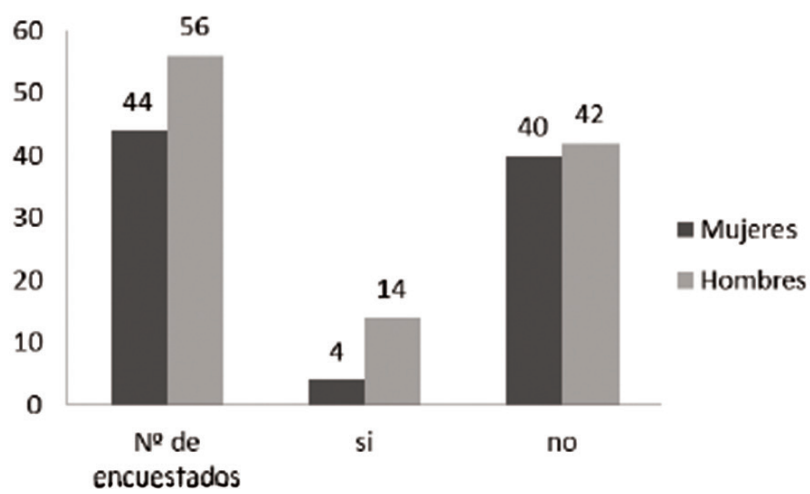

Figura 6. DAP según género de los encuestados.

Con respecto a los intervalos de edad obtenidos por medio del estudio, se encontró que el intervalo que muestra mayor disponibilidad a pagar es aquel entre los 30 - 39 años de edad con 8 personas $(28.5 \%$ del intervalo) dispuestas a pagar por la restauración, seguido del intervalo de edad de más de 50 años, con 4 personas $(22.2 \%$ del intervalo) dispuestas a pagar por la restauración ecológica, estando en 3 lugar el intervalo de edad entre 40-49 años con 4 personas (11.7\% del intervalo),y finalmente el intervalo entre 20-29 años ninguna persona mostro disposición a pagar por la restauración ecológica (Figura 7). 


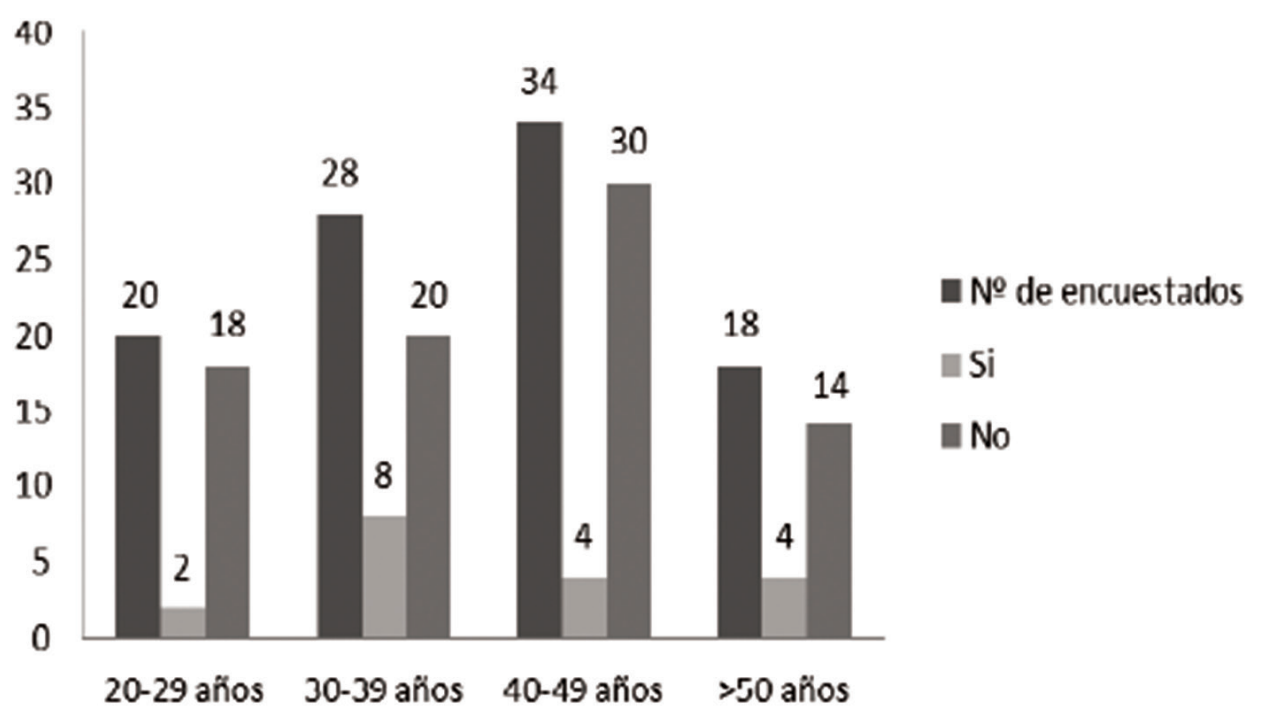

Figura 7. DAP por intervalo de edad.

La disposición a pagar frente a la variable número de hijos, en la cual se demuestra que de los encuestados aquellos que presentan la mayor disponibilidad a pagar por la restauracion son los cuales tienen un solo hijo, dentro de esta categoria se encontraron 24 personas de las cuales 12 es decir el $50 \%$ de la categoria, mostraron una dispocision a pagar, seguido de este aquellos con 2 hijos los cuales 6 (30\% de la categoria) evidencian que estarian dispuestos a pagar, posteriormente se encuentran los datos de los que presentan 3 hijos, de estos 2 personas (6.25\% de la categoria) estan dispuestos a pagar, y finalmente aquellos con 4 hijos ninguno mostro una disponibilidad a pagar (Figura 8).

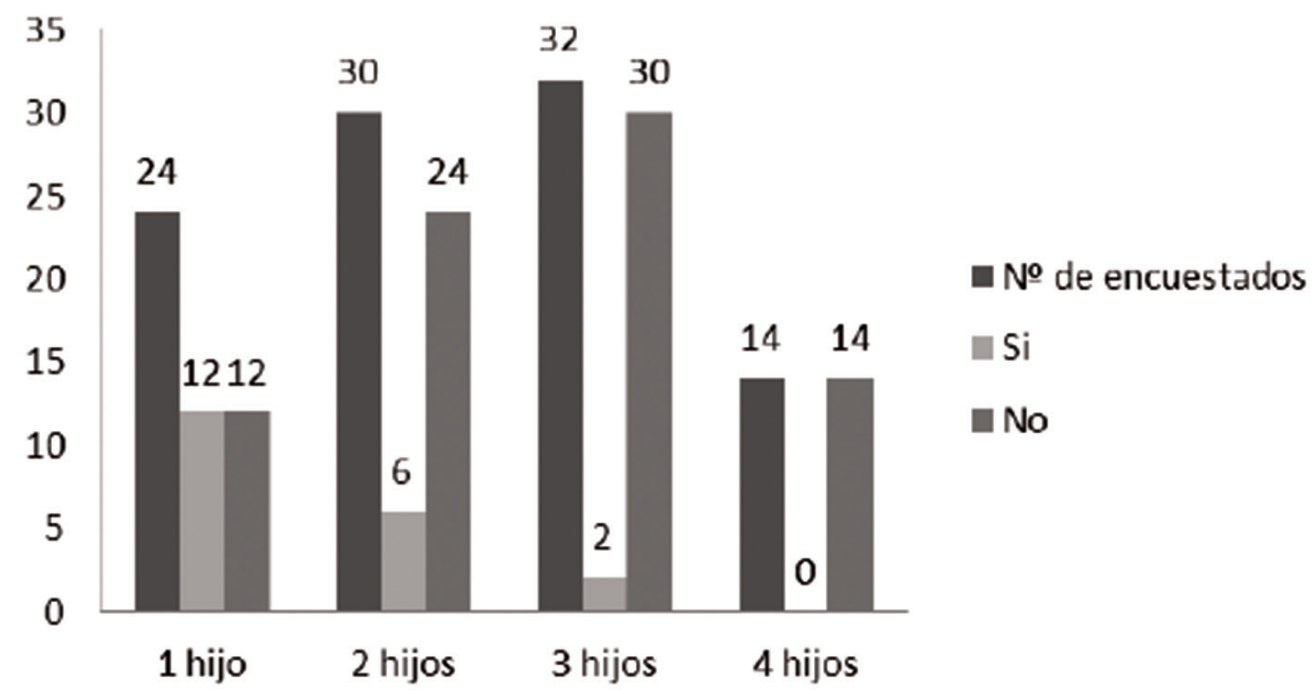

Figura 8. DAP frente a numero de hijos. 
En la Figura 9 se muestra la relacion entre el nivel de escolaridad y la disposicion a pagar por la restauracion ecologica de la cantera Cueva del zorro. Donde se evidencia que el nivel de escolaridad con la mayor proporcion de las personas dispuestas a pagar es aquella con estudios tecnicos, de las cuales 4 de las 4 estan dispuestas a pagar, seguido de aquellas con bachillerato de las cuales 10 de las $36(27.7 \%)$ respondieron positivamente a la disponibilidad a pagar, en cuanto a aquellos con estudios de primaria 4 de los 44 encuestados (9\%) muestran una disponibilidad a pagar, en ultimo lugar se ubican las personas que no tienen estudios las cuales ninguna presenta una disponibilidad a pagar.

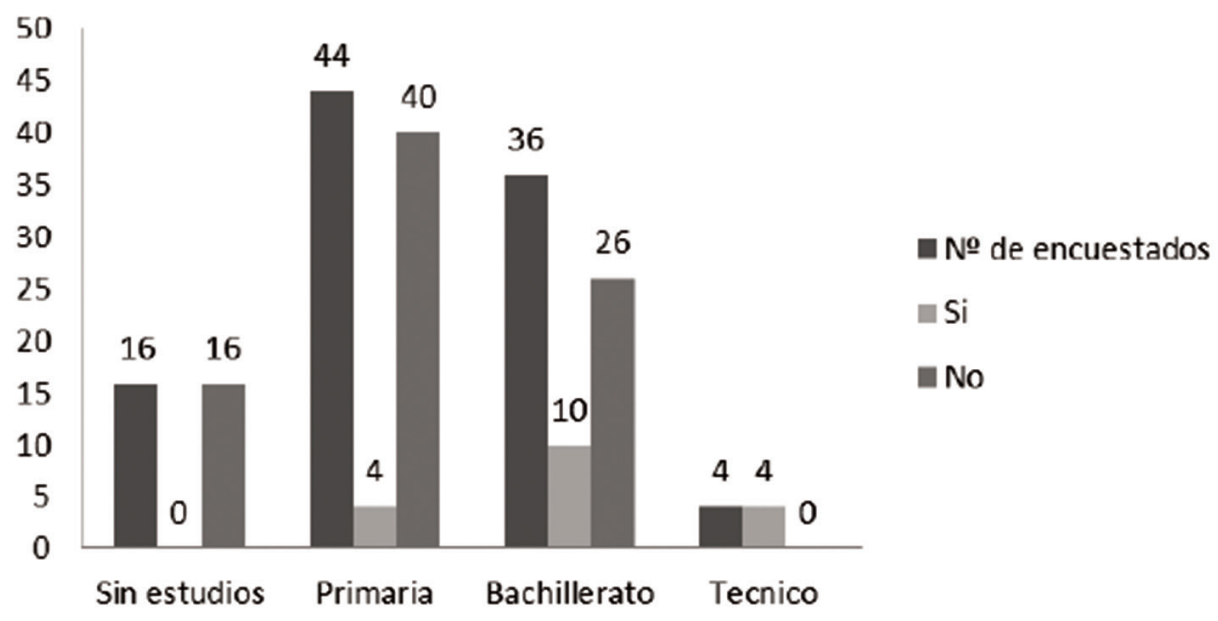

Figura 9. DAP frente a nivel de escolaridad.

En la Figura 10 se muestra el caso del ingreso familiar relacionado a la disponibilidad a pagar en donde el intervalo con mayor disponibilidad a pagar es las personas que tienen ingresos con mas de 800 mil pesos, evidenciando que 6 de las 6 estan dispuestas a pagar, para las personas que presentan ingresos familiares entre 700-799 mil de los 24, 8 (33.3\%) estan dispuestos a pagar, seguido de las personas que tienen ingresos familiares entre 600-699 mil de los cuales solo 4 personas (28.5\%) contestaron positivamente respecto a la disponibilidad a pagar. Para los rangos entre 300-399 mil, 400-499 mil y 500-599 mil ninguno de los encuestados mostró que estuviera dispuesto a pagar por la restauracion ecologica de la cantera Cueva del zorro.

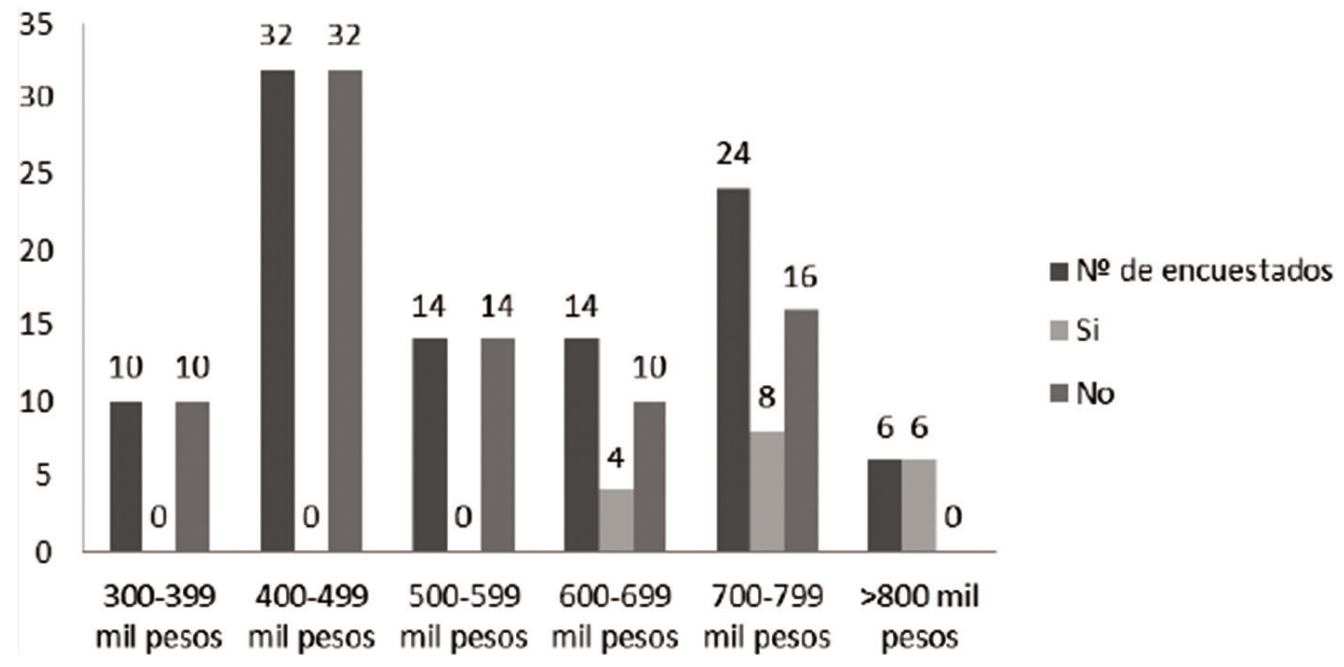

Figura 10. DAP por ingreso familiar. 
En general, los datos obtenidos de las encuestas cuando a la población evaluada se le presenta el caso hipotético de tener que pagar por realizar la restauración ecológica de la cantera Cueva del zorro en el municipio de Soacha. De lo cual resulto: $82 \%$ de las personas no pagarían ningún valor, seguido del
$2 \%$ los cuales estarían dispuestos a pagar 1.000 pesos para realizar la restauración. El valor que sigue es el de 2.000 pesos para lo cual 6 personas se mostraron dispuestas a pagar, el $8 \%$ estarían dispuestas a pagar 3.000 pesos y el $2 \%$ restante mostro disponibilidad a pagar 5.000 pesos (Figura 11).

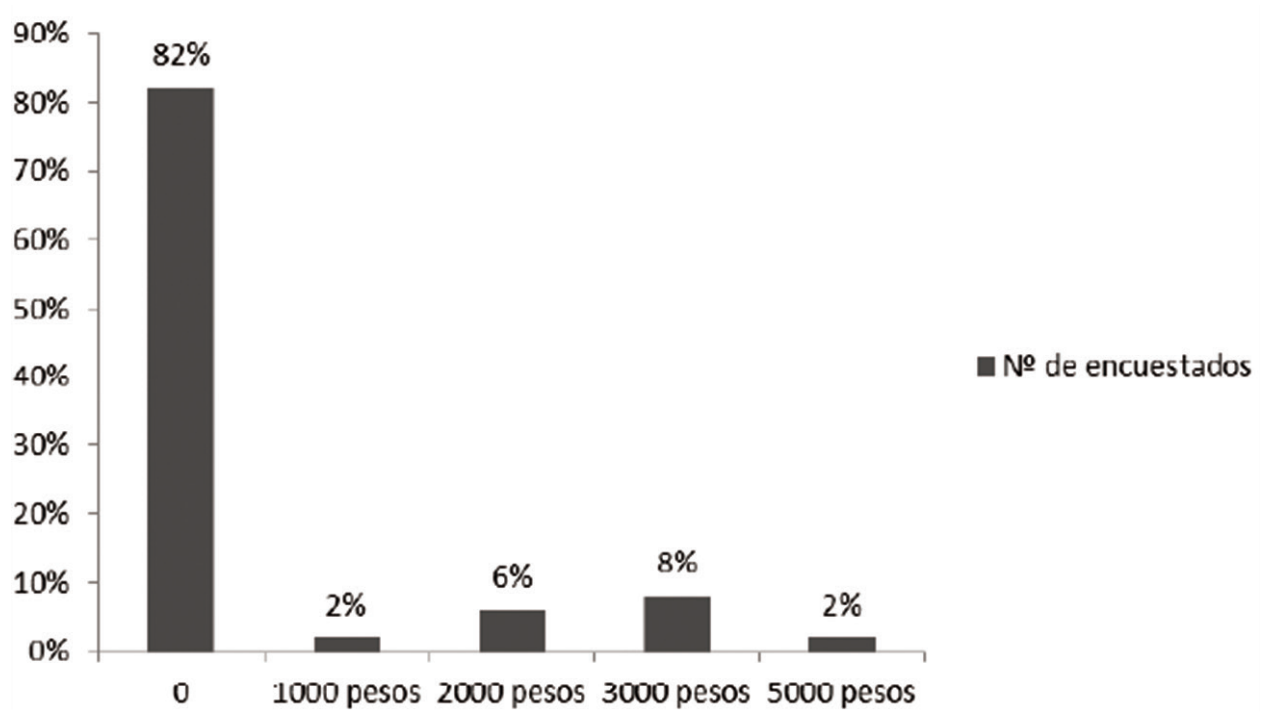

Figura 11. DAP de los encuestados.

\section{Discusión}

De acuerdo con los resultados obtenidos, se evidencia en la comunidad de la vereda Panamá del municipio de Soacha que el medio ambiente para la gran mayoría de la población encuestada es de gran importancia; esto, debido principalmente al hecho que se encuentran ubicados en un área rural. Consecuente con lo anteriormente mencionado la población encuestada demostró un gran interés por la restauración y recuperación de las áreas degradas por la actividad minera en la cantera Cueva del zorro, ya que como puede verse en los resultados es la actividad que consideran más dañina y perjudicial para la calidad ambiental de la zona.

Contrario a la importancia que se le otorga al ambiente, la necesidad de restauración evidenciada por la comunidad y la consideración de la actividad minera como uno de los mayores detonantes de la degradación ambiental. Esta investigación revelo una falta de integración de la comunidad hacia el pago de un impuesto destinado a la realización de la restauración ecológica en las áreas degradadas. Esta información se puede evidenciar en los resultados de las encuestas, los cuales demuestran que solo 18 de las 100 personas encuestadas mostraron estar dispuestos a pagar por dicha restauración; lo cual es muy bajo para una comunidad que aun viéndose afectada por la minería de la cantera Cueva del zorro y con la posibilidad de cambiar el panorama en cuanto al mejoramiento en la calidad ambiental y calidad de vida, deciden no hacerlo porque les implica un gasto monetario adicional.

La disponibilidad a pagar se vio afectada por diferentes variables socioeconómicas; en primera medida se observó que claramente está condicionada por el nivel de ingresos, el número de hijos y el nivel de escolaridad, aunque también se ve una clara tendencia con respecto a los intervalos de edad y al género. 
La DAP disponibilidad a pagar es directamente proporcional al nivel de ingresos, de acuerdo con los resultados obtenidos, mostrándose que las personas que están dispuestas a pagar el mayor monto son además las que mayor ingreso mensual obtiene; mientras que aquellas que obtienen menos del mínimo no están dispuestas a pagar. Esto debido a que viven en una comunidad de estrato 1, bajo-bajo, ubicada en el municipio de Soacha, cuyo nivel de desempleo es muy alto (Alcaldía de Soacha, 2012), y los cuales cuentan con muy pocos recursos económicos; por lo tanto viéndose influenciados por su condición socio-económica al momento de dar un juicio de valor con respecto a la encuesta realizada en el estudio; y aun presentando una conciencia respecto a la necesidad de la restauración de la cantera Cueva del zorro y respecto a los perjuicios ambientales y en términos de salud que esta actividad genera, no presentan la posibilidad de pagar por lo anteriormente mencionado.

Según la mayor parte de los encuestados, la responsabilidad de la restauración es única y exclusiva de la empresa generadora de los daños, y no de los particulares. Lo anterior se relaciona directamente con el concepto de free-rider, problema del polizón, de acuerdo con el cual, las personas no se responsabilizan por el pago del cuidado de los bienes de uso común, ya que esperan que estos costos sean asumidos por otros, aun si el beneficio es colectivo, debido a que el pago es individual (Olsson, 1965).

De acuerdo con lo mencionado anteriormente acerca del tema del polizón, gran parte de la población, se debe hacer un énfasis en el monto -valor dado, valor ofrecido a pagar- de la disponibilidad a pagar, ya que aquellos que afirmaron tener una disposición a pagar por la restauración ecológica seleccionaron en su gran mayoría los valores opcionales de la encuesta más bajos.

El nivel de escolaridad demostró ser influyente; al igual que en el caso del ingreso mensual familiar, la disponibilidad a pagar mostro ser directamente proporcional al nivel de estudios de los encuestados, ya que en los niveles educativos más altos se presentó la mayor proporción de personas dispuestas a pagar por la restauración, evidente en el caso de los técnicos, de los cuales el 100\% afirmó estar dispuesto a realizar el pago; a medida que este nivel desciende, esta disponibilidad a pagar se reduce, llegando al caso de aquellos encuestados sin estudios, cuya disposición a pagar es nula. Esto comprueba lo mencionado por Sepúlveda (2011), ya que al tener mayor educación, presentan mayores conocimientos y responsabilidad hacia el ambiente.

Para el caso del número de hijos presentado por parte de los encuestados, se encontró en cambio, a diferencia de los casos anteriores una condición inversamente proporcional al momento de la disposición a pagar, ya que como pudo ser visto en los resultados del estudio entre menor sea la cantidad de hijos por familia estos presentan una disposición a pagar mayor.

En cuanto a la relación de género y disponibilidad a pagar no se demostró interés por parte de las mujeres, ya que 44 mujeres encuestadas solo 4 dijeron estar dispuestas a dar un impuesto (caso hipotético) por la restauración ecológica de la cantera cueva del zorro, esto se puede ser debido al hecho que en su mayoría las encuestadas son madre cabeza de familia, por lo cual consideran el pago por la restauración, un costo extra que no pueden afrontar dadas sus condiciones socioeconómicas. Esto también puede ser producto del nivel de estudio de las encuestadas, debido a que los 4 técnicos son hombres, y la mayor parte de los que tienen estudios medios también son de este género, por lo tanto se puede evidenciar con este estudio como la falta de educación en el género femenino condiciona radicalmente su visión al medio ambiente, mientras que los hombres presentan un mayor nivel de educación en la gran mayoría de los encuestados razón por la cual hay un mayor número de encuestados de este género que presentan disposición a pagar.

El comportamiento de los encuestados de la vereda panamá en el municipio de Soacha frente a la restauración ecológica de la cantera cueva del zorro, se acerca al homo economicus (interés individual) alejándose totalmente de interés social (homo reciprocantes) el cual busca el beneficio social (Gintis, 2000). 
Según Ostrom (1990) en donde se afirma que en toda sociedad siempre va a existir alguna o algunas personas que actúan como gorrones los cuales buscan beneficiarse de los aportes que realizan los demás, en este caso los encuestados no están dispuestos a realizar un pago por la restauración ecológica pero esperan que otros lo hagan por ellos aun viendo beneficiados por la restauración ecológica.

\section{Conclusiones}

La condición socioeconómica de las personas es directamente influyente en la toma de decisiones que involucren un componente monetario.

La disponibilidad a pagar por parte de la comunidad de la vereda panamá por la restauración ecológica de la cantera Cueva del zorro no es significativa, debido a que de 100 encuestados solo 18 mostraron interés por pagar un impuesto adicional para la realización de esta actividad, lo cual genera preocupación ya que las personas aun viéndose afectadas en términos de salud y calidad de vida por la actividad minera, ofreciéndoles la opción de pagar por mejorar esta condición, caso hipotético, muestran una preferencia por salvaguardar su dinero e invertirlo en lo que ellos consideran de mayor importancia como lo es el alimento o los servicios entre otros.

La valoración ambiental no es significativa en el estudio de poblaciones con niveles socieconomicos bajos, ya que aun si estas personas tuvieran el interés por pagar cierta cantidad de dinero, no podrían hacerlo por la condición en la que se encuentran.

Es importante realizar programas de educativos para la vereda Panamá en el municipio de Soacha, debido a que se demostró que el nivel educativo de las personas incide directamente en la toma de decisiones hacia el medio ambiente.

Es necesario buscar alternativas en la valoración contingente del ambiente, para el caso de comunidades de escasos recursos, ya que los resultados obtenidos con técnicas tradicionales producen grandes sesgos debido a que no son precisos.

\section{Literatura citada}

1. Alcaldía de Soacha (s.f). Documentos sobre el municipio. Sector minero. Recuperado de: http://www. soacha-cundinamarca.gov.co/index.shtml?apc=v-xx1$\& \mathrm{x}=1439698$

2. Alcaldía de Soacha (2012). Recuperado de: http:// www.soacha- cundinamarca.gov.co/informacion_general.shtml

3. DANE (2003). Censo experimental de Soacha. Comunicado de prensa. 13 junio 2003, Recuperado de: http://www.dane.gov.co/files/prensa/comunicados/ cp_censo_experim_soacha4.pdf

4. De la Rosa, A. (2008). Bioestadística. México: manual moderno.

5. Downie, N. \& Heath, R. (1983). Metodos estadísticos aplicados. México: editorial HARLA.

6. Fedesarrollo (2008). La minería en Colombia: Impacto socioeconómico y fiscal. Recuperado de: http://www. fedesarrollo.org.co/wp-content/uploads/2011/08/Laminer\%C3\%ADa-en-Colombia-Informe-de-FedesarroIlo-2008.pdf

7. Grajales, P. (s.f.). Valoración contingente del impacto ambiental de la construcción de la infraestructura vial del proyecto hidroeléctrico Porce III: aplicación a las microcuencas del área de influencia directa. Recuperado de http://www.bdigital.unal.edu.co/5607/1/paulaandreagrajales.2005.pdf

8. Gintis, H. (2000). Beyond homo economicus: evidence from experimental economics. Ecological Economics. 35(3): 311-322.

9. Kristrom, B. \& Riera, P. (1997). El método de la valoración contingente. Aplicaciones al medio rural español. Economía Agraria, 179: 133-166.

10. Milgrom, T. (2007). Environmental aspects of rehabilitating abandoned quarries: Israel as a case study. Landscape and Urban Planning, 87(3): 172-179.

11. Ministerio de Ambiente y Desarrollo Sostenible (2012). Plan Nacional de Restauracion: restauracion ecologica, rehabilitacion y recuperacion de areas disturbadas. República de Colombia.

12. Olson, M. (1965). The logic of collective action, $2^{\underline{a}}$ ed., Cambridge: Harvard University Press, 1971 [La lógica de la acción colectiva, México: Limusa, 1992].

13. Ostrom, E. (1990) Governing the commons: the evolution of institutions for collective action. Cambridge; New York: Cambridge University Press.

14. Rozo, N. (2007). Efecto de la aplicación de mezclas de biosólidos y estériles sobre las primeras etapas de la sucesión en la Antigua arenera Juan Rey. Bogota-DC. (tesis de pregrado). Pontificia Univerisdad Javeriana.

15. Sepúlveda, C. (2011). Análisis de la percepción y disposición a pagar por la Huella de Carbono de leche fluida en consumidores de la ciudad de Valdivia. (tesis de pregrado). Universidad Austral de Chile, Facultad de Ciencias Agrarias. 
16. Singh, A. et al. (2002). Plantations as a tool for mine spoil restoration. Curr. Sci., 82: 1436-1441.

17. Sisben (2013). Base de datos de población sisbenizada de la vereda panamá municipio de Soacha, Cundinamarca.

18. Tomasini, D. (s.f). Valoracion económica del ambiente. Departamento de Economía, Desarrollo y Planeamiento Agrícola. Facultad de Agronomía. Universidad de Buenos Aires, Departamento de Economía, Desarrollo y Planeamiento Agrícola.
19. Vargas, O. \& Reyes, S. (2011). La restauración ecológica en la práctica: memorias I congreso colombiano de restauración ecológica y II simposio nacional de experiencias en restauración ecológica. Universidad Nacional de Colombia, Bogotá, 2011.

20. Vicente, A., Martin, N., James, D., Birss, M., Lefebvre, S. \& Bauer, B (2011). Minería en Colombia ¿a qué precio?. PBI Colombia, 18, 2-47.
Conflicto de Intereses Los autores declaran no tener ningún conflicto de intereses

Recibido: 22 de marzo de 2016 Aceptado: 03 de mayo de 2016 